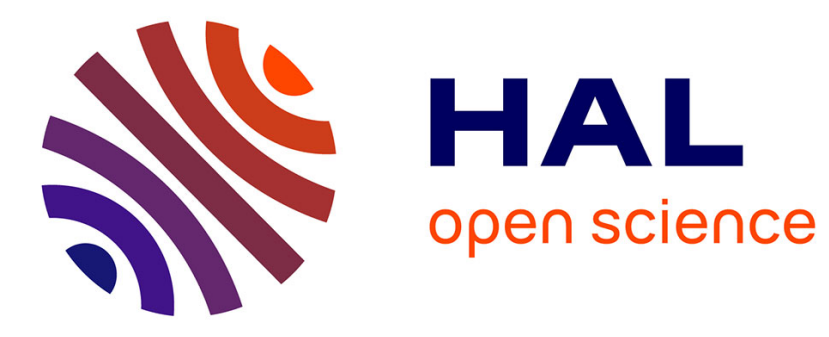

\title{
Extractive SPL adoption applied into a small software company
}

\author{
Luisa Rincón, Edwin Munoz, Juan-C Martinez, Maria-C Pabon, Gloria
}

Alvarez

\section{- To cite this version:}

Luisa Rincón, Edwin Munoz, Juan-C Martinez, Maria-C Pabon, Gloria Alvarez. Extractive SPL adoption applied into a small software company. 2016 XLII Latin American Computing Conference (CLEI), Oct 2016, Valparaíso, Chile. pp.1-8, 10.1109/CLEI.2016.7833388 hal-02302959

\section{HAL Id: hal-02302959 \\ https://hal.science/hal-02302959}

Submitted on 1 Oct 2019

HAL is a multi-disciplinary open access archive for the deposit and dissemination of scientific research documents, whether they are published or not. The documents may come from teaching and research institutions in France or abroad, or from public or private research centers.
L'archive ouverte pluridisciplinaire HAL, est destinée au dépôt et à la diffusion de documents scientifiques de niveau recherche, publiés ou non, émanant des établissements d'enseignement et de recherche français ou étrangers, des laboratoires publics ou privés. 


\title{
Extractive SPL Adoption Applied Into a Small Software Company
}

\author{
Luisa Rincón * ${ }^{*}$, Edwin Muñoz ${ }^{\dagger}$, Juan-C Martinez *‡, Maria-C Pabón * and Gloria Álvarez * \\ *Departamento de Electrónica y Ciencias de la Computación, Pontificia Universidad Javeriana \\ Cali, Colombia \\ $\dagger^{\dagger}$ Acople Tecnológico SAS - Cali - Colombia \\ $\ddagger$ Centre de Recherche en Informatique, Université Panthéon Sorbonne \\ Paris, France
}

\begin{abstract}
Acople Tecnológico SAS is a Colombian enterprise specializing in software development whose products use devices for biometric facial and fingerprint recognition. The functional overlap of such products provides an opportunity for systematic reuse, which reduces software development and maintenance costs. In this paper we report our experiences, lessons learned and future work from applying concepts of software product line engineering to evolve three of its different software applications into a software product line. Based on our experience, we explain how we have tailored existing concepts of product line engineering, specifically how we have followed the process thus far, which includes the variability analysis that refers to the study of commonalities and variabilities among a set of software applications, the development of reusable software assets, and the derivation of individual products from the product line.
\end{abstract}

Keywords-Software Product Lines, Domain Engineering, Feature Models, Core Assets, Industrial Case, Reusable artifacts, Extractive adoption

\section{INTRODUCTION}

Software Product Line Engineering (SPLE) is a software development paradigm that seeks to create product families rather than creating individual products. These families group a set of products that share common characteristics while having some variations from each other. The software product lines (SPL) allow to capitalize on the development effort in many products while efficiently managing their differences. The SPL promise benefits such as reuse and reduction in development time and costs [1].

The domain engineering and the application engineering are the two main process of the SPLE [1]. Domain engineering consists of studying the commonalities and variabilities among a set of software applications and developing the reusable artifacts and resources that form the basis for the software product line (core assets) ${ }^{1}$. These core assets will enable the rapid construction of particular software system belonging to the same family of products. Application engineering, in turn, consists of analyzing, designing, building, customizing, and testing the final products, using the core assets and the specific requirements expressed by the customers. The domain engineering process is executed once in order to construct the

\footnotetext{
${ }^{1}$ http://www.sei.cmu.edu/productlines/start/glossary/index.cfm

978-1-5090-1633-4/16/\$31.00 (C)2016 IEEE
}

product-line infrastructure, whereas the application engineering phase is executed each time a new product is derived from the product line.

SPLE has been applied in several domains, especially in large-scale software development [1]-[6], but it also has increasingly garnered interest from small to medium-sized companies. Motivated by those experiences our general goal is to apply the related concepts of software product line engineering to Acople Tecnológico SAS, a small Colombian enterprise specializing in software development.

In this paper, we propose a step to achieve this goal. Specifically, we present how we have followed the process thus far, which includes the variability analysis that refers to the study of commonalities and variabilities among a set of software applications, the development of reusable software assets and the derivation of individual products from the product line. We have followed an extractive adoption strategy [7], because it allows us to develop the new assets while taking advantage of the software artifacts already developed by Acople Tecnológico. In fact, we used as reference three applications that Acople Tecnológico had previously launched on the market.

We believe the use of a SPLE approach in Acople Tecnológico can be significantly advantageous because this company has a portfolio of software products that share a significant amount of functionality and code, but are developed, tested and maintained separately. This has led to high costs in terms of time and technical resources which wasted technical resources and involved a high investment in time and cost.

The remaining parts of this paper are structured as follows. Section II, gives an overview of the characteristics of the company and its context (domain analysis) and presents the model as a result of analyzing company's applications. Section III explains how we defined the reusable modules that implement the features defined in the variability model that we proposed as a result of analyzing the company. Section IV, presents the benefits and lessons learned in the use of a SPLE approach. Section V, provides a summary of works related to our proposal, and finally Section VI presents the conclusions and suggests future research directions. 


\section{VARIABILITY ANALYSIS}

\section{A. Domain Analysis}

Acople Tecnológico SAS is a small software company located in Cali, Colombia. It has over six years of experience and since its establishment, it has been delivering software solutions at the enterprise level using biometric devices. Over the years, Acople Tecnológico SAS has developed and marketed several independent applications. In fact, well-known Colombian enterprises such as Proservis Temporales SAS, Coca Cola Cali, or Servientrega SA, have successfully used Acople Tecnológico's applications.

Based on the company's experience, we have chosen three applications developed by Acople Tecnológico that share many different features as a base to carry out an analysis of their variability to define the variability inherent to the product line.

Chosen applications were: Working Time Control, Access Control, and Restaurant. A brief explanation of each one is presented below:

- Working Time Control: this application measures how much time each employee works during his working day. Its main purpose is to identify the start and the end of the working days to prevent fraud and irregularities when working time of each employee is calculated.

- Access Control: it manages permissions to control access to highly secure areas. To achieve this, the application identify who each person is by means of biometric devices.

- Restaurant: this application uses biometric identification to control the schedule to deliver food and to authorize people for receiving lunches or dinners when they are sponsored by an enterprise.

Several analysis techniques can be used to analyze and model the variability. In our case, we used feature modeling as the notation to represent our product line, because this notation has been widely adopted in the Software Product Lines community [8]. Under this notation, each feature is a node in a tree structure and represents a distinguishable characteristic that is relevant to our stakeholders, from a technical or functional point of view. The tree's root of the Feature Model represents whole product line, and it is decomposed into its most prominent features. Then, these prominent features are decomposed into their prominent sub-features, and so on, until the family is entirely decomposed into features.

Among features there are several relationships to define how features are related each other, depending on, for instance, if a child is an optional or a mandatory feature. Specifically, there are three types of relations: mandatory, optional and group cardinality. Mandatory features (dark circles) are always selected, optional features (white circles), can be chosen or not, and cardinality group features (arc with UML cardinality), where the selection is realized among a limited set of alternatives according to the cardinality. In addition, the notation introduces two types of constraints among features: requires and excludes. The requires constraint states that for a given feature to be selected, the required feature has to be selected too. The excludes constraint states that for a given feature to be selected, the excluded feature has to be deselected [9].

Given the complexity of each application, we modeled the variability of each application through individual feature models first. Doing so, we identified features shared by the three applications such as Reports, People, Notification or Device. Furthermore, we identify features such as Calendar or Schedule that were considered only by one of the applications, but could be optionally used in other applications to extend their functionality. Moreover, we detected some features that were managed as separate concepts, but could be generalized into more general terms in order to unify them as one. For instance, we created the feature Application Type to generalize the three types of applications that were factored to extract the product line, and we decomposed this feature into three subfeatures: Working Time, Restaurant and Access.

\section{B. Resulting Feature Model}

Figure 1 shows the feature model we constructed as a result of analyzing each application separately. Below, we briefly outline the functionality of each feature.

- Application Type refers to the main functionality of a product. Currently, it includes Working Time, Access, and Restaurant types. There is a mandatory relation between the features Working Time and Schedule. In fact, a product that includes Working Time should also include Schedule, to manage staff shifts.

- People is related, on the one hand, with the management of the people that could be identified by the biometric devices (Person Identification) and, on the other hand, with the management of the administrative users (Application Users). Any product of this product line should always identify, by means of biometric devices, people who belong to the organization (Insiders), and it may optionally identify people not belonging to the organization (Outsiders).The application administrative users could generate Reports.

- Records represents the mechanism used to transfer the information captured by the biometric devices to the application. This communication could be Online when the device has Internet access or Batch when records should be manually downloaded to the system. It is noteworthy that if the Application Type is Access, then it is essential to have Internet access, and thus there is an exclusion relation between the features Access and Batch.

- Device refers to different kind of biometric devices that could be used by an application. Currently, the SPL considers two kinds of devices, Facial and Fingerprint, however, the SPL could be extended to incorporate iris, voice, or card recognition.

- Number of People represents the maximum number of people that the biometric device could identify. This feature has two sub-features $P O$ to 1200 , which means from zero up to one thousand two hundred people is supported by the device, and $P 1200$ to infinite, which means the device supports over one thousand two hundred people. Currently, the facial 


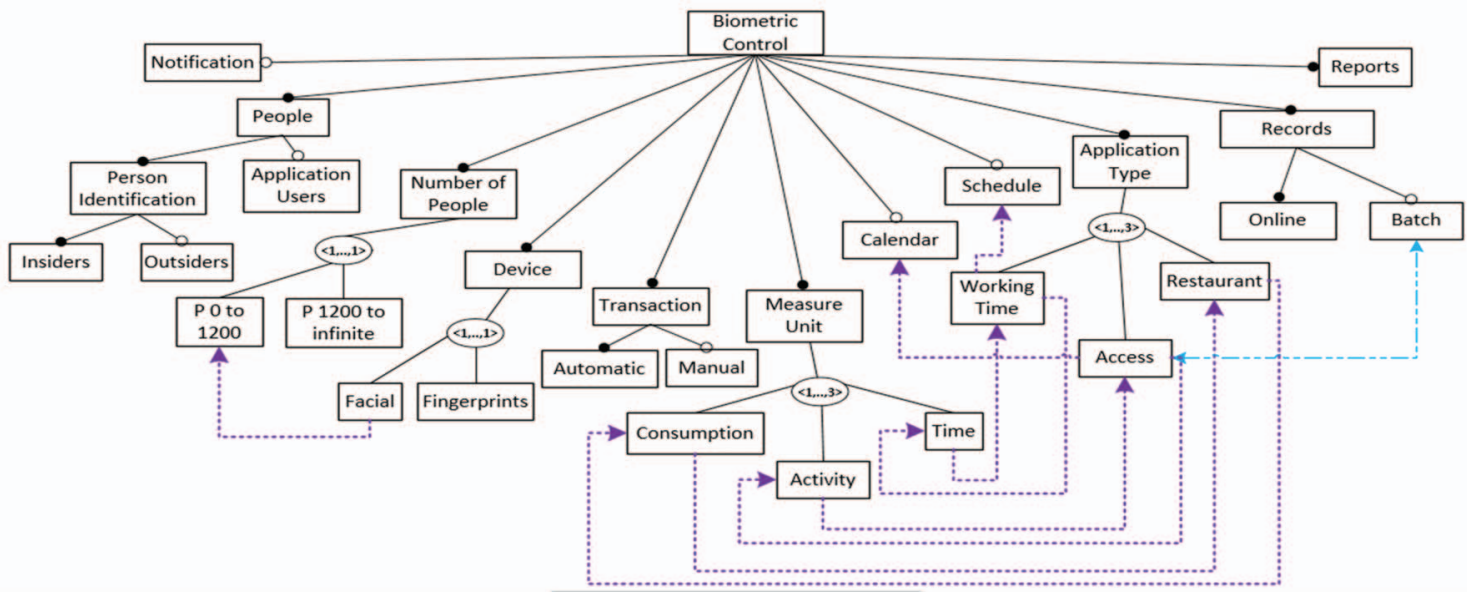

Figure 1: Feature model which represents the variability and commonality in the analyzed domain

recognition devices incorporated in the SPL support a maximum of 1200 people. Thus, there is a requires relation between the features Facial and P 0 to 1200 .

- $\quad$ Transaction points out how a new event is registered. An automatic transaction takes the data directly from the biometric device. Otherwise, the data comes from a manual process. Any product derived from the SPL would have defined one transaction type. Optionally, a biometric application can manually register data. Otherwise, data should be captured by biometric devices.

- Measure Unit indicates what is measured, calculated, or controlled by the application. For example, the feature Consumption has to be used for product or service management, or to control the consumption. Activity has to be used in transactional applications, for example, to manage the purchasing of goods and services. Time has a variety of uses, such as, working or delivery time calculation. Depending on what type of application is selected it is necessary to select a different Measure Unit. We use among those features some requires relations, as can be seen in the Figure 1 , to control this dependency.

- Notification is about notifying application users and stakeholders of particular system conditions or events.

- Calendar includes the organization and management of calendar, including holidays. According to our domain analysis, applications that contain the feature Access must also include the Calendar feature.

The feature model is the result of an iterative design process with the participation of a functional and technical expert from Acople Tecnológico. This expert, who has many years of experience, validated the resulting model assuring that we had correctly modeled the business domain concepts. Moreover, we verified the model by using VariaMoses, a modeling tool that offers automatic verification of feature models. It helped us to be sure that our model is correct since it is free of errors. Additionally, by using Variamos,

\footnotetext{
${ }^{2}$ http://variamos.com/
}

we found the number of different products that could be obtained from our feature model. Variamos determines the number of products based on the combinations resulting from adding and subtracting features. The analysis of the proposed feature model established that 320 different products could be generated. Taking into account that the model includes the features found in three previously developed products, the possibility to obtain 320 products shows that software product lines facilitate reuse planning.

According to Acople Tecnológico's experience, of these 320 potential applications, the feature Application Type determines the main commercial products and therefore six of them, resulting from combining this feature and its sub features, are very attractive from a commercial point of view, even though other possible combinations could be commercialized too. Based on this idea, Acople Tecnológico plans to define a classification of products according to the Application Type to manage a licensing schema. This schema could include several versions, like Basic, Business, and Corporate licenses. The license would be defined along with the product characteristics and functionality. For example, a Working Time control product could be a Basic application whereas a Working time + Access + Restaurant product could has a Corporate license.

Once we analyzed the common and variable elements in our domain, the next step was to design a flexible software architecture that supports the specified variations. The next section explains how this goal has been achieved in our particular case.

\section{DeVelopment of ReUSAble ASSETS}

At the current level of the proposed product line, we have defined software modules as core assets of our product line, but we have not yet defined other assets such as unitary test cases, process descriptions, or any other element of a software production process. In this section, we explain how we have defined reusable modules that implement the features defined in our variability model, and how we used those modules when we want to assemble products from the product line. 


\section{A. Core Assets Definition}

According to Bachmann and Bass [10], there are two possible techniques to implement a variation on core assets: module replacement and data controlled variation. By using the first technique, multiple code-based versions of a particular module are implemented and later, at the execution time or at an earlier stage, the correct one is chosen. In the second technique, the variability is internally implemented inside of a single module, hiding the adaptation on the architecture level.

Given that Acople Tecnológico was just becoming with the product line approach, we considered more suitable to group features with close functionality in modules and to implement the variability inside the module when necessary. In total, we defined nine modules: People, Devices, Schedule, Calendar, Records, Application Type, Reports, Notifications, and Common. Calendar, Schedule and Notifications are modules with an optional scope [11], which means they may or may not be included in the applications obtained from the product line. Modules Devices and Application Type have an open scope [11], which means they have already known variants, but a set of possible values can still be extended later at the time, for instance by adding a new type of application. Modules People and Records have a binary scope because they have two possible variants which are already known [11], and the rest of the modules are reusable software components that implement the product line commonality, but they do not support variability. At an internal level, we use a table to keep the traceability between each module and the features that it implements. Next, we explain the main aim of each module.

The People module manages operations related to the features People and Application Users. Some of those operations are to manage user accounts, permissions, log-offs, password changes, and more. On the other hand, the functionality of the Person Identification feature is controlled by recording each person directly on the biometric device.

The Device module controls the communication between the software application and the biometric devices by means of operations for downloading records, controlling the number of users, and adding new biometric devices. This module supports the feature Device. In addition, this module also supports the features, Number of People, $P 0$ to 1200 and $P 1200$ to infinite which are useful to define how many people will be supported by the software application.

The Schedule module is in charge of managing aspects related to staff scheduling and the Calendar module manages information about holidays required by the applications. The Records module handles the registry of data delivered by the biometric devices as well as data required by each application type. This module also enables the addition of attributes to the Records table according to the particular requirements of each application. The attributes are added trough SQL statements, like ALTER TABLE, and the portions of code that manipulate the database information manage those schema differences, making them transparent to the final user. This module implements the features Records and Transactions, and their sub-features.

The module Application Type supports the three types considered in the SPL and the measure units associated with each type. The implementation of this module consists of two parts: an interface that exposes services shared by all the application types, and a particular java class for each application type that includes its own functions exposed as services. The Notifications module has the functionality for composing and sending messages and supports the feature Notification. Finally, the Common module supports functions shared among all the modules such as exceptions and dates handling, validations, currency format, and internationalization.

\section{B. Core Assets Design and Implementation}

We decided to develop assets by using Java EE technologies with the aim to quickly change Acople Tecnologico's conventional software development into a software product line engineering approach. We decided to develop assets by using Java EE technologies since previous assets were developed on Java EE technologies too, and therefore we can reuse, as much as possible, previous assets. To make this possible in an ordered way, each module presented in the Section III-A was implemented as a distinct (physically separated) code unit. This strategy, called compositional approach [12], provides us a direct link between each feature and its implementation, giving us a high degree of feature traceability and therefore easier maintenance over time [13].

We used feature model as a tool for modeling the domain of interest, but from a detailed design point of view, we used UML for describing, at the design level, the specific details of each module. As each module was considered as an independent part of code, we followed the Model View Controller pattern to design and implement each one of them. We chose this architecture because it gives us independence between the user interface of each module and its business logic. For instance, Figure 2 shows the design that we have made for the devices module. At the right side of the figure, into the devices model package, we put the business entities and we marked with blue color the ones that are not defined by this module, but that are required by it. Into the DevicesBusinessController package, we define the main business concepts. By using welldefined interfaces with clearly exposed properties and events, as presented by Gacek [14], we support variability for two different type of devices: fingerprint and facial recognition. However, the SPL is open to adding new types of devices in the future, as long as they follow the defined interface. Finally, on the right side, we present the graphic user interfaces (views) required to provide the full functionality of this module.

Model and controller layers were implemented as a Java SE project, which were packaged as a jar files, whereas the view layer was separated into two different parts, a view controller (managed bean) and a folder with .xhtml files that represents the module screens. As a result we had for each module three assets: a .jar file, a folder with .xhtml files and a .class file.

Model layer was implemented using the Data Access Object Pattern (DAO), to separate low-level data accessing operations from high-level business services. Common to all modules we defined and implemented a generic interface $I D A O<T>$ where we declare standard database operations. Then, we defined the model entities that each module needed and we implemented a specific DAO for each entity. We reuse the most of the model entities previously developed by 


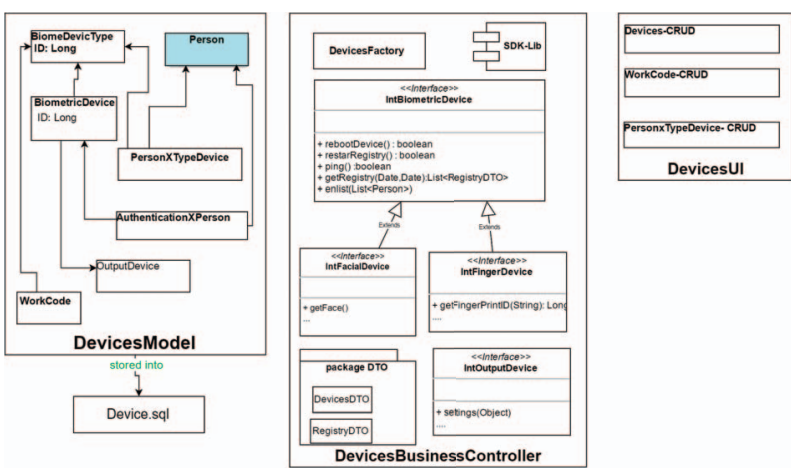

Figure 2: Detail design module Devices

Acople Tecnológico, although we created custom annotations to add meta-data into the Java source code to facilitate data validations using reflection techniques.

For the controller layer, we defined a public java interface with the available services of each module and at least one Java class for implementing those services. Outside of those modules only the public operations were accessible, obtaining as a result high cohesion and low coupling among modules.

Finally, the view layer was implemented with the Java Server Faces - JSF- technology. This framework provides the file faces-config.xml which is useful to define the managed bean associated to each view and the navigation rules among the different views of each module. For instance, according to the business logic in the module Devices it is possible to gain access to the view createDevice.xhtml only from the view listDevices.xhtml. For each module we defined those rules to incorporate them into a general faces-config file when a new application is derived. We also created a general template file template.xhtml which defines the appearance and distribution (header, content and footer) for all the final user screens.

\section{Generate product line members}

Currently, feature selection and product derivation are both manual activities. This means that although many parts of the implementation have been prepared during the domain implementation and can be reused, some activities are done manually. For instance, it should be defined what features will be selected from the feature model. Also, it has to be solved the relation between modules and features to determine which modules will be used according to the features selection, and each product has to be manually assembled based on the reusable artifacts. Later, the resulting product is verified before being delivered to a customer by functional test cases. We plan in the future to elaborate automated unit tests to automatize this part.

We based the derivation of new members of the product line on the "Configuration" [15] approach. In this approach, the first version of the product is based on a consistent set of modules that can function together. In this sense, to facilitate the derivation we created a base for any new application by creating a new web project in Eclipse JEE IDE ${ }^{3}$. This project

\footnotetext{
${ }^{3}$ https://eclipse.org/downloads/packages/eclipse-ide-java-eedevelopers/mars2
}

respects the structure presented in Figure 3. Then, we added to this project all the developed modules with the exception of the optional ones (see Notifications, Calendar, and Schedule modules ). As a result, we obtain a set of assets which not conform a full executable application because options and settings in the binary and open modules are still open at the module level, but are very useful to simplify the product derivation. In this sense, by first defining the common aspects between the products instead of specifying their differences, we give a basic reference frame, but we still allow freedom in product specific architectural deviation.

In an effort to take advantage of the Acople Tecnológico's experience all applications obtained from the product line will follow the rules defined by the Java Servlet Specification, and the general architecture presented in Figure 3. The product line architecture is a layered architecture based on the client - server paradigm. Business, Data and presentation layers are separated into different source files.

Specifically, the configuration and derivation tasks that have to be done manually to complete the product are the following:

1) Create a new database in the database engine installed on the application server.

2) Select from the feature model those choices that define client requirements and satisfy the model restrictions.

3) Identify the modules that have to be included according to the features previously selected, or configure modules previously included if they implement full mandatory features.

4) Execute the SQL statements of each selected module to create tables and populate them with their basic data when required.

5) Move into the LIB folder the .jar files of each module previously identified and other third-party libraries required by the application.

6) Implement the requirements that could not be satisfied through the reuse of modules previously developed and adapt artifacts such as screens, style-sheets and figures to satisfy the customer requirements.

7) Copy into the sub folder classes, located in the WEBINF folder, files with .class extension. These files are the view controllers which implement the logic associated to the views of each selected module.

8) Modify into the folder classes the file datasource.properties to adjust the database name for the new application.

9) Create in the xhtml folder a sub folder for each selected module and copy there the corresponding .xhtml files. Other screens that do not allow to any specific module, but are required by the application should be included here as well.

10) Generate the distributable file that constitutes the web application (war extension).

11) Configure the application server with the connection string and the jdbc driver according to the database engine. The connection name must match with the name defined in the datasource.properties file.

12) Deploy the application (.war file) in the application server. 


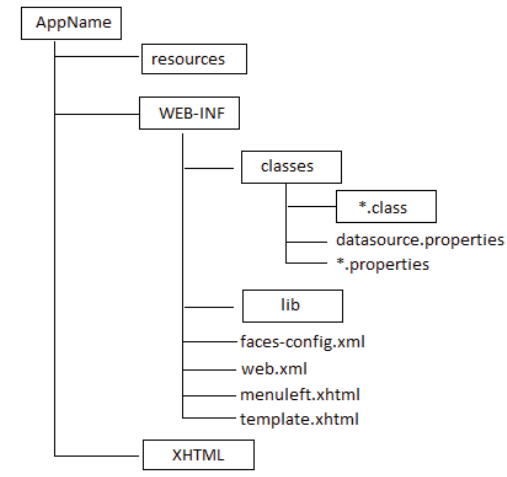

Figure 3: General Java EE architecture defined for the product line members

As a result of following those steps, a functional web application is available to prepare it for final delivery to the customer. Then functional tests are required to ensure that this product satisfies customer requirements.

\section{DISCUSSION}

The development of the SPL for applications based on biometric devices could bring several benefits to Acople Tecnológico, in addition to the several lessons that have been learned from the process of designing and development the SPL. In this section both of them are discussed.

\section{A. Benefits for the Business}

Acople Tecnológico changed its methods to build applications by applying a development paradigm with better results in development time and quality. In fact, given the need of Acople Tecnólogico to get to market fast, the old versions of the three products had evolved without much focus on design. As a consequence, it considered a lot of copy and paste of code and had many lines of code providing the same functionality but written in a different way. After follow the product line approach, all the developed core assets offer more functionality that their pre-product line counterparts with a reduction in the number of lines of code. This improvement is very useful considering that more lines of code would represent more troubles, especially during the maintenance phase. Another secondary benefit was the uniform look and feel and code style adopted after the full re-factor required in this process, considering that both aspects contribute to improve the final product quality.

Furthermore, the SPL could be used to extend the portfolio of Acople Tecnológico's applications in the future, allowing them to envision new applications since different contexts could require the same functionality. Taking advantage of this opportunity, Acople Tecnológico already generated a new product targeted to preschool institutions, allowing them to register persons authorized to pick up each child and to identify who picked them each day. In this way, the school increases the security in the process of releasing the children to their families. This new product has many similarities with the Working Time one, and its set of features is one of the 320 combinations produced by the Variamos analysis.
In the same way, the SPL eases the creation of any of the three products including particular functionality required by a specific client. This was the case of a new variation of the Working Time application. Before the implementation of SPL methodologies, a particular request was attended by duplicating the application code — in this case, Working Timeand making further modifications over it. With the SPL, the basic application functionality is coded in libraries that are added to a new project, then specific functionalities are treated as new features, generating new libraries to implement them. Eventually, those new features could be included in the features model being available to use on new types of products.

Moreover, with the aim to facilitate the inclusion of new features or application types, the modules design, and implementation involved extension points. As an example, the component Marks has attributes to identify the application type and extra attributes to manage diverse data types (numeric, alphanumeric, or boolean).

Thus far, the SPL also facilitated the generation of a third new product that integrates the functionality of Working Time, Restaurant, and Access applications. With this product Acople Tecnológico served one client that wanted to use the three products. Before the SPL implementation, three different applications would have been sold and installed.

\section{B. Lessons Learned}

Several lessons have been learned during the analysis, design, and implementation of the SPL, among them, are the following ones.

The features model allowed us to abstract the problems and to focus development efforts on reusing artifacts, leading to high quality and functionality, reducing the development time and giving quicker customer response. This improves the company's business opportunity.

The variability model was useful to identify applications' modules. It includes business rules that guarantee high cohesion and low coupling, easing the reuse of components.

The modules' dependencies definition was crucial to avoid circular references and to achieve low coupling. Besides dependencies point out the components' implementation order. Moreover, as far as possible, a product derived from the SPL will contain only the logic associated with the features the product comprises.

Even though in our particular case the 320 feature combinations could be analyzed to select a set of new products that could bring the Acople Tecnológico's market attention, generally that is not a viable task due to the vast number of possible combinations. However, having the combinations is valuable since it allows us to verify if a particular set of product requirements is valid in the SPL. In fact, when a new client wants a product from the company, Acople Tecnológico can easily identify what requirements are covered by the product line and therefore could better estimate the cost and the development time required to satisfy them. If there are requirements that cannot be satisfied through the reuse of assets previously developed into the product line, the company negotiates and develops those requirements as a product-specific addition. 
The development of an SPL in an SME could bring some advantages. Among them, the products are small like the organization itself, in the sense that they are composed of few components. This facilitates the analysis, design, and development stages, as well as the transition from the methodologies applied before to the new ones. However, there are also some disadvantages; the main one is that the organization has few human resources. Thus, maintaining the operation along with the development of the SPL is a demanding task.

Finally, the most complex task was to design the modules, maintaining the abstraction while increasing reusing. The experience of Acople Tecnológico in attending the biometric applications market was a key aspect in the definition of generic modules. The design facilitated the codification task since it provides a better comprehension of components' functionality. Thus, since benefits are greater than the cost of design and implementation of the SPL, Acople Tecnológico is planning to develop, in the near future, an SPL for the second set of products in their portfolio, related with auto service portals directed to employees, clients, and providers, among others.

\section{RELATED WORKS}

In recent years the industry has reported several experiences regarding the implementation of Software Product Lines. The Product Line Hall of Fame 4 lists the best SPL industrial applications presented in the Software Product Line Conference over the years. Among them, Siemens Healthcare [4] combined SPL with agile development to overcome some challenges detected in the development of imaging products. Those products, ranging from medical scanners to visualization systems, share medical imaging functionality, including different image modalities, data types, analysis algorithms and manipulation concepts. The U.S. Army Live Training Transformation and the U.S. Navy command and control systems of Naval surface combatants [3] are two SPLs that bring improvements in quality, time to deployment, cost, and engineering productivity.

Linden et al. [16] collect the experiences of eight companies, including Nokia, Bosch, Philips and Siemens, describing the main effects of the SPL adoption. Additionally, Koziolek et al. [5] report the experiences from executing domain and economic analysis in more than 20 software systems belonging to four application cases in the ABB engineering company and concluded that SPL should be applied in only one of the four cases. They identified feature domains, subdomains and components with the potential to constitute a SPL. Pohl et al. [1] present another fifteen successful implementations. The authors highlight the costs and development time reduction, as well as the improvement of product quality. Birk et al. [2] analyzed the SPL practices used by five organizations, among them Hewlett-Packard and Bosch. Laguna y Hernández [17] report the application of methods and techniques of the Product Line Engineering in the domain of e-commerce software and describe the modeling, configuration, design and implementation stages. Sanchez et al. [18] report the development of an SPL to generate e-learning web mining products that include variations of three major features: e-learning platform, queries, and data mining suite. The authors describe the analysis of the

\footnotetext{
${ }^{4}$ http://splc.net/fame.html
}

domain and the application engineering processes. Parra et al. [6] describe the implementation of SPLs in Heinsohn Business Technology. They developed a SOA modular architecture to support the adoption of a SPL over a set of JEE artifacts already developed for different domain applications.

Even though SPL has been applied in several domains, most studies refer to projects carried out in large companies. In contrast, in this paper, we report the experience and lessons learned from the SPL implementation in a small organization. Besides, different to other cases, in this work we adopted an extractive approach that took advantage of the Acople Tecnológico experience in the market. Moreover, even though biometric recognition is included as a feature in many SLP examples [19], [20], to the best of our knowledge, there is no report of an SPL including biometric devices for authentication with an aim different to software access control. The software products obtained by the SLP presented in this paper use the authentication for other purposes, such as working time or restaurant consumption control.

\section{CONCLUSIONS AND FUtURE WORK}

This paper has presented how a set of products that use biometric devices have been re-factored, using an extractive approach, into a Software Product Line. The crucial aim of introducing the product line approach in Acople Tecnológico was to improve reuse in a planned way, which means a reduction in the time and cost of developing new related products.

The approach developed in this paper presents the experiences and lessons learned from applying SPLE in a particular industrial setting. Doing so, we define a variability model expressed as a feature model that was inspired by a real case, and therefore may be used as a reference for future researches. Furthermore, we explain our general strategy to develop reusable components based on a compositional approach and the benefits and lessons learned from applying software product lines into an small enterprise.

So far we have come a long way in the implementation of our product line, but we are aware that there are other steps to follow in order to finish our goal. As a next step, we will continue extending our proposal. We aim to define a strategy for configuring and assembling as automatic as we can the modules that we have developed, which will allow us to create new products from the product line. We have considered tools as Gradle or Maven to automatize the assemble process. Moreover, we are interested in incorporate non-functional requirements such as security and availability into the SPL development to improve the current software assets.

\section{ACKNOWLEDGMENTS}

This work has been partially supported by the Pontificia Universidad Javeriana Cali, Colombia, under the program COFINPRO2014 which sponsors the Project No. 4127.

\section{REFERENCES}

[1] K. Pohl, G. Böckle, and F. J. van Der Linden, Software Product Line Engineering: Foundations, Principles and Techniques. Springer-Verlag New York, Inc., 2005 
[2] A. Birk, G. Heller, I. John, K. Schmid, T. von der Massen, and K. Muller, "Product line engineering, the state of the practice," Software, IEEE, vol. 20, no. 6, pp. 52-60, Nov 2003.

[3] P. Clements, S. Gregg, C. Krueger, J. Lanman, J. Rivera, R. Scharadin, J. Shepherd, and A. Winkler, "Second generation product line engineering takes hold in the dod," The Journal of Defense Software Engineering, USAF Software Technology Support Center, 2013.

[4] M. Kircher and P. Hofman, "Combining systematic reuse with agile development: Experience report," in Proceedings of the 16th International Software Product Line Conference - Volume 1, ser. SPLC '12. New York, NY, USA: ACM, 2012, pp. 215-219.

[5] H. Koziolek, T. Goldschmidt, T. de Gooijer, D. Domis, S. Sehestedt, T. Gamer, and M. Aleksy, "Assessing software product line potential: an exploratory industrial case study," Empirical Software Engineering, pp. 1-38, 2015.

[6] C. Parra, D. Joya, L. Giral, and A. Infante, "An soa approach for automating software product line adoption," in Proceedings of the 29th Annual ACM Symposium on Applied Computing, ser. SAC '14. New York, NY, USA: ACM, 2014, pp. 1231-1238.

[7] C. W. Krueger, "Easing the Transition to Software Mass Customization," in Revised Papers from the 4th International Workshop on Software Product-Family Engineering, ser. PFE '01. London, UK, UK: Springer-Verlag, 2002, pp. 282-293.

[8] T. Berger, D. Lettner, J. Rubin, P. Grünbacher, A. Silva, M. Becker, M. Chechik, and K. Czarnecki, "What is a Feature?: A Qualitative Study of Features in Industrial Software Product Lines," in Proceedings of the 19th International Conference on Software Product Line, ser. SPLC '15. New York, NY, USA: ACM, 2015, pp. 16-25. [Online] Available: http://doi.acm.org/10.1145/2791060.2791108

[9] K. Czarnecki, S. Helsen, and U. Eisenecker, "Formalizing cardinalitybased feature models and their specialization," Software Process: Improvement and Practice, vol. 10, no. 1, pp. 7-29, 2005.

[10] F. Bachmann and L. Bass, "Managing variability in software architectures," ACM SIGSOFT Software Engineering Notes, vol. 26, pp. 126-
132, 2001.

[11] S. D. Kim, J. S. Her, and S. H. Chang, "A theoretical foundation of variability in component-based development," Information and Software Technology, vol. 47, no. 10, pp. 663-673, 2005.

[12] C. Kastner, S. Trujillo, and S. Apel, "Visualizing software product line variabilities in source code," Proceedings of the 2nd International Workshop on Visualisation in Software Product Line Engineering (ViSPLE 2008), collocated with the 12th International Software Product Line Conference (SPLC 2008), 2008.

[13] B. Zhang, S. Duszynski, M. Becker, and B. Zhang, "Variability Mechanisms and Lessons Learned in Practice," in Proceedings of the 1st International Workshop on Variability and Complexity in Software Design, 2016.

[14] C. Gacek and M. Anastasopoules, "Implementing product line variabilities," pp. 109-117, 2001.

[15] S. Deelstra, M. Sinnema, and J. Bosch, "Product derivation in software product families: a case study," Journal of Systems and Software, vol. 74, no. 2, pp. 173-194, 12005.

[16] F. J. v. d. Linden, K. Schmid, and E. Rommes, Software Product Lines in Action: The Best Industrial Practice in Product Line Engineering. Springer-Verlag New York, Inc., 2007.

[17] M. a. Laguna and C. Hernández, "A software product line approach for e-commerce systems," in Proceedings - IEEE International Conference on E-Business Engineering, ICEBE 2010, 2010, pp. 230-235.

[18] P. Sanchez, D. Garcia-Saiz, and M. Zorrilla, "Software product line engineering for e-learning applications: A case study," in Computers in Education (SIIE), 2012 International Symposium on, Oct 2012, pp. $1-6$.

[19] T. E. Fægri and S. Hallsteinsen, "A software product line reference architecture for security," in Software Product Lines, T. Käkölä and J. C. Dueñas, Eds. Springer Berlin Heidelberg, 2006, pp. 275-326.

[20] K. C. Kang, V. Sugumaran, and S. Park, Applied Software Product Line Engineering, 1st ed. CRC press, 2010. 